\title{
THE TIGHT-BINDING APPROACH TO THE DIELECTRIC RESPONSE IN THE MULTIBAND SYSTEMS
}

\author{
P. Županović \\ Department of Physics, Faculty of Science and Art, University of Split, Teslina 10, \\ 58000 Split, Croatia \\ A. Bjeliš and S. Barišić \\ Department of Physics, Faculty of Science, University of Zagreb, P.O.B. 162, \\ 41001 Zagreb, Croatia
}

\begin{abstract}
Starting from the random phase approximation for the weakly coupled multiband tightly-bounded electron systems, we calculate the dielectric matrix in terms of intraband and interband transitions. The advantages of this representation with respect to the usual plane-wave decomposition are pointed out. The analysis becomes particularly transparent in the long wavelength limit, after performing the multipole expansion of bare Coulomb matrix elements. For illustration, the collective modes and the macroscopic dielectric function for a general cubic lattice are derived. It is shown that the dielectric instability in conducting narrow band systems proceeds by a common softening of one transverse and one longitudinal mode. Furthermore, the self-polarization corrections which appear in the macroscopic dielectric function for finite band sys-
\end{abstract}


tems, are identified as a combined effect of intra-atomic exchange interactions between electrons sitting in different orbitals and a finite inter-atomic tunneling.

PACS: $71.10 .+\mathrm{x}, 71.45 . \mathrm{Gm}$

Key words: random phase approximation, dielectric matrix, collective modes, selfpolarization corrections 


\section{Introduction}

Random phase approximation (RPA) is the well-known textbook method [1] for treating the dielectric properties of the weakly interacting electron liquids. Unfortunately the widely used [2 - 5] extension of this method to the multiband systems leads to a cumbersome problem, even when limited only to the calculation of collective modes of the tightly bound electrons in the optical regime. In the present text we propose therefore a new formulation of RPA which leads to a more convenient description of the dielectric response of the tightly bound electrons. In particular it greatly simplifies the analysis of the collective modes giving a direct insight into their physical origin. It also

resolves some long lasting controversies [2, 6, 7] on the local field effects in multiband insulators and metals.

The paper is organized as follows. In Sec.2 we formulate the problem and derive the tight-binding expression for the dielectric matrix. In Sec.3 the determinant of this matrix for the lattice with the cubic symmetry is explicitly calculated in the long wavelength limit in which one can make the multipole expansion of the long range contributions to the bare Coulomb matrix elements. The collective modes and the macroscopic dielectric function which follow from this result are considered in Secs. 4 and 5 respectively. The latter Section also contains the discussion of the so-called self polarization corrections. Concluding remarks are given in Sec.6. 


\section{Dielectric matrix in the tight binding approach}

In the standard approach [2, 3] one uses the plane wave representation and starts from the infinite dielectric matrix $\varepsilon_{\mathrm{G}, \mathrm{G}}{ }^{\prime}(\mathbf{q}, \omega)$ with $\mathbf{G}$ and $\mathbf{G}^{\prime}$ denoting all the vectors of the reciprocal lattice. In order to determine the collective modes and the macroscopic dielectric function $\varepsilon_{M}^{-1}=\left(\varepsilon^{-1}\right)_{00}$ one has to calculate the determinant of the dielectric matrix $\varepsilon_{\mathrm{G}, \mathrm{G}^{\prime}}$. Unfortunately there was no straightforward way to reduce the dimension of this determinant even when only a finite number of presumably relevant tightly bound bands was taken into account. The reason is that the plane wave basis used for the matrix elements of the Coulomb interaction is not suitable for the description of the tightly bound electronic band states. As a consequence, already in the extreme long wavelength limit the analysis of the dielectric properties was rather complicated [2], while sophisticated and physically nontransparent numerical algorithms were inevitable for more complex situations 四, 可.

One easily appreciates that the tight binding (TB) Wannier-like representation with explicit band indices is more appropriate than the plane wave one. Having in mind the fact that the basis defined by band indices and the values of wave vector within the first Brillouin zone brings in the full crystal symmetry (including the discrete translations) like the basis defined in the extended zone scheme, we introduce in the present work the dielectric matrix in the former basis, without any reference to the plane wave indices G. This approach was already used in studying the dielectric properties of the single metallic band [8], but not, to the best of our knowledge, for multiband systems. In 
fact, the TB basis was frequently used indirectly [2, 3, 6, 0, 91, $i . e$. in the calculation of the RPA multiband polarizabilities which appear in the matrix elements $\varepsilon_{\mathrm{G}, \mathrm{G}}{ }^{\prime}$. This led to some simplifications in inverting the dielectric matrix $\varepsilon_{\mathrm{G}, \mathrm{G}}{ }^{\prime}(\mathbf{q}, \omega)$ [10]. We point out below some crucial advantages of the present direct TB representation.

We start by calculating the mean values of the components

$$
\rho_{l l^{\prime}}(\mathbf{q}, t) \equiv 2 \sum_{\mathbf{k}}<a_{l}^{+}(\mathbf{k}, t) a_{l^{\prime}}(\mathbf{k}+\mathbf{q}, t)>
$$

of the density response to the corresponding components of the external potential $V^{e x t}(\mathbf{r}, t)$,

$$
V_{l l^{\prime}}^{e x t}(\mathbf{q}, t)=\frac{1}{N} \sum_{\mathbf{R}} e^{i \mathbf{q R}} \int d^{3} r \varphi_{l}^{*}(\mathbf{r}-\mathbf{R}) V^{e x t}(\mathbf{r}, t) \varphi_{l^{\prime}}(\mathbf{r}-\mathbf{R}) .
$$

Here $\varphi_{l}(\mathbf{r}-\mathbf{R})$ is the TB orbital for the $l$-th band at the $\mathbf{R}$-th site, and $a_{l}^{+}(\mathbf{k})$ is the creation operator for the corresponding Bloch state. The present discussion is limited to the weak coupling in which the bare intraband and interband Coulomb interactions are smaller than the bandwidths and the interband energy differences respectively. Starting from the equations of motion for the operators of the electron-hole pairs $a_{l}^{+}(\mathbf{k}) a_{l^{\prime}}(\mathbf{k}+\mathbf{q})$, and performing the standard RPA steps [1, 11], one arrives at the system of linear equations

$$
\sum_{r}\left(\delta_{p r}-V_{r p} \Pi_{p}\right) \rho_{r}=\Pi_{p} V_{p}^{e x t}
$$

The indices in the eq.(3) stay for the ordered pairs of band indices, $p=\left(l, l^{\prime}\right)$ and denote the transitions between the tight-binding bands. Note that the spin indices can be omitted in the RPA. The function $\Pi_{p}(\mathbf{q}, \omega)$ is the RPA polarization diagram

$$
\Pi_{p}(\mathbf{q}, \omega)=\frac{2}{N} \sum_{\mathbf{k}} \frac{n_{l^{\prime}}(\mathbf{k})-n_{l}(\mathbf{k}+\mathbf{q})}{\omega-E_{l}(\mathbf{k}+\mathbf{q})+E_{l^{\prime}}(\mathbf{k})+i \eta}
$$


with the occupation and dispersion of the $l$-th band given by $n_{l}$ and $E_{l}$ respectively. Evidently, $\Pi_{p}$ is finite only for transitions between (partially) full and (partially) empty bands as well as for the transitions within the partially filled bands.

$V_{p r}(\mathbf{q})$ in eq.(3) is the matrix element of the bare Coulomb interaction in which we keep only the contributions with two and two TB orbitals centered on same crystal sites (two center integrals),

$$
V_{p r}(\mathbf{q})=\sum_{\mathbf{R}} e^{i \mathbf{q R}} \int d^{3} r \int d^{3} r^{\prime} \varphi_{l_{1}}^{*}(\mathbf{r}-\mathbf{R}) \varphi_{l_{2}}^{*}\left(\mathbf{r}^{\prime}\right) \frac{e^{2}}{\left|\mathbf{r}-\mathbf{r}^{\prime}\right|} \varphi_{l_{1}^{\prime}}(\mathbf{r}-\mathbf{R}) \varphi_{l_{2}^{\prime}}\left(\mathbf{r}^{\prime}\right),
$$

with $p=\left(l_{1}, l_{1}^{\prime}\right)$ and $r=\left(l_{2}, l_{2}^{\prime}\right)$. This usual TB approximation which greatly simplifies the system of equations (3i) and works best for long wavelengths [8], is based on the assumption that the overlaps between the orbitals on the neighboring sites are so small that the corresponding contributions to the Coulomb matrix elements are negligible with respect to the contributions retained in the eq.(5)). Basically, this is the only restriction on the present RPA approach. In return it leads to simple $p, r$ selection rules, as will be discussed below. Further on, in the long wavelength limit it will be sufficient to keep only the leading contributions in the multipole expansion of the twosite $(\mathbf{R} \neq 0)$ terms in eq.(5) . The on-site $(\mathbf{R}=0)$ terms are usually omitted, or at least not treated explicitly in the weak coupling limit. However, as it will be argued below, some of these terms are relevant for the understanding of the local field effects.

In the present approach the dielectric matrix is given by the coefficients on the left-hand side of eq.(河),

$$
\varepsilon_{p, r}(\mathbf{q}, \omega)=\delta_{p, r}-V_{r p} \Pi_{p},
$$

in accordance with the definition which follows from the Dyson equation for the 
screened Coulomb interaction [13], and with the standard definition for the special single band case [1].

With the wave vector $\mathbf{q}$ restricted to the first Brillouin zone and with $l$ covering all band indices, the matrix $\varepsilon_{p, r}(\mathbf{q}, \omega)$ represents, like the matrix $\varepsilon_{\mathrm{G}, \mathrm{G}}{ }^{\prime}(\mathbf{q}, \omega)$, a complete RPA dielectric response, with all local field effects taken into account. Strictly, its dimension is infinite, due to the infinite number of possible interband transitions with $\Pi_{p} \neq 0$. However, an approximation which neglects interband transitions with small polarizations $\Pi_{p}$ [e.g. those from the deep (core) orbitals and those to very high empty bands], reduces the dimension of $\varepsilon_{p, r}(\mathbf{q}, \omega)$ to a finite value, equal to the number of retained interband and intraband transitions. This type of simplification with the explicit physical justification cannot be achieved in the plane wave representation which requires numerical truncations in both band and reciprocal lattice indices 幽, 5, 12.

\section{$3 \quad$ Long wavelength dielectric response of the cubic lattice}

The further advantage of the dielectric matrix in the TB representation becomes apparent in the explicit long wavelength limit. For the sake of definitiveness let us consider the cubic lattice and at first take into account only the two-site terms in the eq.(5). An analogous procedure can be carried out for any crystal symmetry. We distinguish the following three types of dominant lattice sums [14 in the multipole expansion of the various matrix elements $V_{p r}(\mathbf{q} \rightarrow 0)$.

For both $p$ and $r$ representing intraband transitions [i.e. the transitions within 
partially filled bands, with $p=\left(l_{1}, l_{1}\right)$ and $\left.r=\left(l_{2}, l_{2}\right)\right]$, the dominant contribution is the monopole-monopole one, given by 15

$$
V_{p r}(\mathbf{q})=\frac{4 \pi e^{2}}{a^{3} q^{2}},
$$

where $a$ is the lattice constant.

If both indices represent interband transitions [i.e. $p=\left(l_{1}, l_{1}^{\prime}\right), r=\left(l_{2}, l_{2}^{\prime}\right)$ with $l_{1} \neq l_{1}^{\prime}$ and $l_{2} \neq l_{2}^{\prime}$ ] the lowest possible multipole contribution is the dipole-dipole term

$$
V_{p r}=\frac{8 \pi}{3} \frac{\mu_{p \|} \mu_{r \|}}{a^{3}}-\frac{4 \pi}{3} \frac{\boldsymbol{\mu}_{p \perp} \boldsymbol{\mu}_{r \perp}}{a^{3}} .
$$

Here

$$
\boldsymbol{\mu}_{p}=e \int d \mathrm{r}^{3} \varphi_{l}^{*}(\mathbf{r}) \mathbf{r} \varphi_{l^{\prime}}(\mathbf{r})
$$

is the dipole matrix element for the transition from the $l$-th to the $l^{\prime}$-th local function, and $\mu_{p \|}$ and $\boldsymbol{\mu}_{p \perp}$ are its respective projections parallelly and perpendicularly to the wave vector.

The third type of matrix elements are those with one interband and one intraband transition [i.e. $p=\left(l_{1}, l_{1}^{\prime}\right)$ with $l_{1}, \neq l_{1}^{\prime}$ and $r=\left(l_{2}, l_{2}\right)$, or vice versa] for which the lowest multipole contribution is of the monopole-dipole form,

$$
V_{p r}=-\frac{4 \pi i e}{a^{3}} \frac{\mu_{p \|}}{q} .
$$

We emphasize that the long-range part of the Coulomb interaction contributes only to the above three types of matrix elements in the long wavelength limit $\mathbf{q} \rightarrow 0$. The other terms in the multipole expansions are higher orders in components of $\mathbf{q}$, and as such are vanishing in this limit. They are therefore irrelevant even if $e . g$. the dipolar factors in the eqs.(8) and (10) are absent for symmetry reasons. 
Let us now divide all transitions into four sets, taking into account particular properties of the cubic symmetry. Each dipolar matrix element (9) is directed along one of three fourfold rotation axes $(\mathrm{x}, \mathrm{y}$ and $\mathrm{z})$ [16]. All interband (dipolar) transitions can be therefore divided into three sets, $\left\{p_{j}\right\}$, where $j=1,2$, and 3 stay for $x, y$ and $z$ axis respectively. The fourth set $\left\{p_{0}\right\}$ contains all (at most three [15]) intraband (monopolar) transitions. Furthermore, for each dipolar matrix element $\boldsymbol{\mu}_{p_{1}}$ there are two other matrix elements $\boldsymbol{\mu}_{p_{2}}$ and $\boldsymbol{\mu}_{p_{3}}$ with the same absolute value, which complete a three-dimensional irreducible representation of the cubic point group [16].

These symmetry properties, together with the factorized form of the matrix elements (8) and (10), reduce the system of eqs.(3) to four linear equations. To this end we introduce the following linear combinations of the density components $\rho_{p}$ belonging to the four sets defined above:

$$
\rho_{0}=\sum_{p \in\left\{p_{0}\right\}} \rho_{p}
$$

and

$$
\rho_{j}=-i \frac{\mathbf{q} \hat{\mathbf{n}}_{j}}{e} \sum_{p \in\left\{p_{j}\right\}} \mu_{p} \rho_{p} \quad j=1,2,3
$$

with $\hat{\mathbf{n}}_{j}$ denoting the unit vector along the $j-t h$ axis. Summing up separately equations (3) with $p \in\left\{p_{0}\right\}$ and $p \in\left\{p_{j}\right\}, j=1,2,3$, one arrives after few straightforward steps to the decoupled systems of two and two equations. The first system represents the longitudinal response

$$
\begin{gathered}
{\left[1+\left(4 \pi \alpha_{c}\right)^{-1}\right] \rho_{0}+\sum_{j=1}^{3} \rho_{j}=-\frac{a^{3} q^{2}}{4 \pi e^{2}} V^{e x t}} \\
\rho_{0}+\left[\frac{2}{3}+\left(4 \pi \alpha_{I}\right)^{-1}\right] \sum_{j=1}^{3} \rho_{j}=i \frac{a^{3}}{4 \pi e} \mathbf{q} \cdot \mathbf{E}_{e x t}
\end{gathered}
$$


while two linearly independent equations among the following three equations

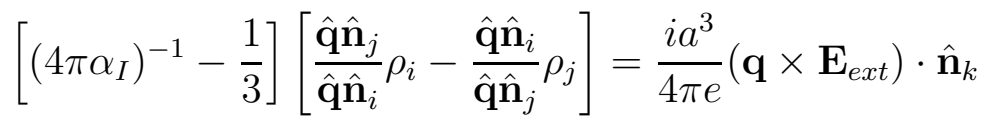

(with $i \neq j \neq k$ ) represent the transverse response. Here $\hat{\mathbf{q}} \equiv \mathbf{q} / q, V^{e x t}(\mathbf{q}, \omega)$ is the external scalar potential and $\mathbf{E}_{\text {ext }}(\mathbf{q}, \omega)$ is the external electric field. Eqs.(15) are slightly generalized with respect to the original eqs.(3), since they allow for the finite transverse electrical field. The intraband and interband polarizabilities are given by

$$
\begin{gathered}
\alpha_{c}=-\frac{e^{2}}{a^{3} q^{2}} \sum_{p \in\left\{p_{0}\right\}} \Pi_{p} \\
\alpha_{I}=-\frac{1}{a^{3}} \sum_{p \in\{I\}}\left|\mu_{p}\right|^{2} \Pi_{p}
\end{gathered}
$$

respectively. $\{I\}$ stands for any of three sets $\left\{p_{j}\right\}, j=1,2,3$.

The above separation to the longitudinal and transverse response does not depend on the direction of $\mathbf{q}$, as it should be for the cubic crystal. Since the original density components $\rho_{p}$ follow from eqs.(3) once the combinations which figure in eqs.(13 - 15) are known, the above procedure gives the explicit solution for the dielectric response of cubic crystals. In particular, since the systems of equations (3) and (13 - 15) are connected by linear transformations and thus have a common determinant, it follows immediately from the latter equations that

$$
\operatorname{det}\left[\varepsilon_{p, r}(\mathbf{q}, \omega)\right]=\varepsilon_{l}\left(\varepsilon_{t}\right)^{2}
$$

with

$$
\varepsilon_{l}=\left(1+4 \pi \alpha_{c}\right)\left(1+\frac{8 \pi \alpha_{I}}{3}\right)-16 \pi^{2} \alpha_{c} \alpha_{I}
$$


and

$$
\varepsilon_{t}=1-\frac{4 \pi \alpha_{I}}{3}
$$

The original determinant of infinite order is reduced so to the algebraic expression in which the infinite summation in $\alpha_{I}$ can be easily truncated due to the explicit physical meaning of the terms in eq.(17).

\section{Collective modes}

The expressions $(18$ - 20) are particularly convenient for the discussion of the collective modes which appear as isolated poles of $\operatorname{det}(\varepsilon)$ in the $\omega$-plane, and of their Landau damping due to the incoherent intraband and interband electron-hole excitations, provided the band dispersions and corresponding TB orbitals are specified. The details of this calculation for simple band models are discussed elsewhere [13]. Here we present the most important conclusions concerning the collective modes.

Besides the obvious case of a single band conductor $\alpha_{C} \neq 0, \alpha_{I}=0$ with intraband plasmons as collective excitations, the result (18) covers also a less evident asymptotic limit of an atomic (molecular) insulator. Namely, after assuming that all interband energies $\left|E_{l}^{\prime}-E_{l}\right| \equiv E_{p}$ are much larger than the corresponding bandwidths, the interband polarizability (17) reads

$$
\alpha_{I}=-\frac{2}{a^{3}} \sum_{p \in\{I\}} \frac{n_{p} E_{p}\left|\mu_{p}\right|^{2}}{\omega^{2}-E_{p}^{2}}
$$

where $n_{p}$ is the number of electrons per a site that take part in $p$-th

transition. Inserting this expression together with $\alpha_{c}=0$ into eqs.(19, 20), one 
gets the dipolar collective modes whose spectrum just coincides with that of Frenkel excitons [17]. It should be noted in this respect that the present RPA result is valid in the weak coupling regime [18]. We remind that the same spectrum is obtained [19] in the opposite strong coupling regime when the bandwidths are negligible with respect to the on-site Coulomb repulsion $V_{p r}(\mathbf{R}=0)$ between two electrons on different orbitals (i. e. the $\mathbf{R}=0$ contribution in the expression (5) with $l_{1}=l_{1}^{\prime}$ and $l_{2}=l_{2}^{\prime}$ ).

The Frenkel excitonic spectrum thus appears to be a common asymptotic limit of the two otherwise incompatible regimes. Indeed, the corrections due to the finite bandwidths are scaled differently in the two regimes, i.e. by $E_{p}$ and $V_{p r}(\mathbf{R}=0)$ respectively. In the strong coupling regime the collective modes are coherent superpositions of atomic electron-hole excitations, gradually delocalized by finite bandwidths. The appropriate representation in the weak coupling regime starts from the electrons in Bloch states. Accordingly, in contrast to the former, the latter regime includes also the narrow band conductors in which the interband polarizability can be still approximated by eq.(21), while the intraband polarizability is finite and given by

$$
4 \pi \alpha_{c}=-\frac{\omega_{p l}^{2}}{\omega^{2}}
$$

where $\omega_{p l}=4 \pi n_{e} e^{2} / m^{*} a^{3}$ is the frequency of the intraband plasmon, and $m^{*}$ and $n_{e}$ are respectively the effective mass and the number of electrons (or holes) in the metallic $\operatorname{band}(\mathrm{s})$.

As can be seen from eq.(19), the finiteness of $\omega_{p l}$ causes the renormalization of the dipolar longitudinal modes, but does not affect the transverse modes (20). This coupling between longitudinal intraband and interband collective modes is to be traced 
back to the finite monopole-dipole interaction (10). Although the transverse modes (20) are not screened by the intraband charge fluctuations, there is an interesting relation between them and the hybridized longitudinal modes of eq.(19). Let the frequency of the lowest transverse mode in eq.(20), $\omega_{t_{l o w}}$, tend to zero. Then the equation $\varepsilon_{l}=0$ has the solution, $\omega_{l_{l o w}}$, which also tends to zero. The reverse is also true. The ratio of two frequencies is

$$
\left(\frac{\omega_{l_{\text {low }}}}{\omega_{t_{\text {low }}}}\right)^{2}=\frac{\omega_{p l}^{2}}{\omega_{p l}^{2}+\frac{4 \pi \alpha_{I}(\omega=0) \prod_{p} E_{p}}{\prod_{j} \omega_{T_{j}}^{2}}}
$$

where $\omega_{T_{j}}$ are frequencies of all other (stable) transverse collective modes. Note that

$\omega_{l_{\text {low }}}<\omega_{t_{\text {low }}}$. The dielectric instabilities in multiband conductors are thus characterized by the simultaneous softening of two collective modes. This somewhat surprising, and to the best of our knowledge new result is the consequence of the proper treatment of the metallic screening of the interband (dipolar) excitations in the present approach. We note that the cubic symmetry chosen here is not essential for the validity of this result [13].

\section{Macroscopic dielectric function}

The macroscopic dielectric function $\varepsilon_{M}$ follows immediately from eqs.(13).14). To this end it suffices to utilize the averaging scheme [3] by which the probe electrical field and the corresponding density response are $\mathbf{E}_{e x t}=i \mathbf{q} V^{e x t}(\mathbf{q}) / e$ and $\rho(\mathbf{q})=\sum_{j=0}^{3} \rho_{j}(\mathbf{q})$ 
respectively. According to the standard definition [1] $\varepsilon_{M}$ is then given by

$$
\varepsilon_{M}(\mathbf{q}, \omega)=\left[1+\frac{\rho(\mathbf{q}, \omega)}{a^{3} q^{2} V^{e x t} / 4 \pi e^{2}}\right]^{-1}=\frac{\varepsilon_{l}}{\varepsilon_{t}}=1+4 \pi \alpha_{c}+\frac{4 \pi \alpha_{I}}{1-\frac{4 \pi}{3} \alpha_{I}}
$$

This expression reproduces some earlier results [2, 3, 6, 7], but, unlike them, does not contain any type of the so-called self-polarization corrections. In our approach these corrections are related to the on-site (intra-atomic) contributions to the bare Coulomb matrix elements (5), not taken into account until now. Obviously, all these contributions are independent of $\mathbf{q}$. Furthermore, it is easy to see that due to the symmetry reasons only those on-site matrix elements $V_{p r}(\mathbf{R}=0)$, with $p$ and $r$ representing the transitions of the same (monopolar, dipolar, etc) type, are finite. On the other side, in the RPA it is sufficient to keep only those on-site terms for which the leading multipole contributions in the corresponding two-site sums are constant or vanish. Thus, the on-site terms which complete the monopole-monopole two-site sums (7) in eq(5) [like e.g. the on-site "electrostatic" repulsions $V_{p r}(\mathbf{R}=0)$ between two electrons sitting at the same or different orbitals] are irrelevant in the limit $\mathbf{q} \rightarrow 0$. Since the on-site contributions which correspond to the monopole-dipole two-site sums (10) vanish, the most interesting on-site contributions are those going together with the next in order ( i.e. dipole-dipole) two-site contributions (8).

The inclusion of those on-site contributions into the expression (2) prevents unfortunately the reduction of the system (3) to the explicitly solvable form (13, 14). Still, some interesting conclusions can be drawn for simple models [13], like that with only two bands $\left(l=l_{1}, l_{2}\right)$ connected by a finite dipole matrix element (9) $\boldsymbol{\mu}_{p}, p=\left(l_{1}, l_{2}\right)$. Then the dipole-dipole matrix element $V_{p, \tilde{p}}$ of eq(8) has to be completed by the on-site 
term

$$
V_{p, \tilde{p}}(R=0)=\int d^{3} r \int d^{3} r^{\prime} \varphi_{l_{1}}^{*}(\mathbf{r}) \varphi_{l_{2}}^{*}\left(\mathbf{r}^{\prime}\right) \frac{e^{2}}{\left|\mathbf{r}-\mathbf{r}^{\prime}\right|} \varphi_{l_{2}}(\mathbf{r}) \varphi_{l_{1}}\left(\mathbf{r}^{\prime}\right)
$$

which has the meaning of the exchange between two atomic orbitals. Furthermore, the interband polarizability (17) reduces to

$$
\alpha_{I}=-\frac{1}{a^{3}}|\mu|^{2}\left(\Pi_{p}+\Pi_{\tilde{p}}\right)
$$

The macroscopic dielectric function $\varepsilon_{M}$ can be still written in the form (24), but with the interband polarizability $\alpha_{I}$ replaced by

$$
\alpha_{I}^{\prime}=\frac{\alpha_{I}}{1-V_{p, \tilde{p}}(R=0)\left(\Pi_{p}+\Pi_{\tilde{p}}\right)} .
$$

Note that there is no effect of this type on the intraband (metallic) polarizability $\alpha_{c}$, in accordance with the already mentioned fact that for small $\mathbf{q}$ the long-range monopolemonopole Coulomb interaction dominates over all on-site "electrostatic" repulsions.

The denominator in the eq.(27) can be simply interpreted as the atomic screening of the interband polarizability $\alpha_{I}$. Since it has itself the RPA form, the formulation (3) apparently treats both, the band and the local (intra-atomic) dielectric responses selfconsistently at the same (RPA) level of approximation.

When the bandwidths are finite, the intra-atomic and the inter-band screenings in eq. (27) cannot be decoupled. As a consequence the result for $\varepsilon_{M}$ deviates from the Lorentz-Lorenz (LL) form in the sense that the effective interband polarizability $\alpha_{I}^{\prime}$ which enters into eq (24) cannot be reduced to the form (17), $i$. e. to the sum of interband polarization diagrams. This deviation is usually named a self-polarization correction, originally derived by Adler [2] within the plane-wave representation. The 
result (27) brings us to the clear microscopic interpretation of this correction, recognized as a combined effect of the intra-atomic screening due to the on-site exchange Coulomb interactions and of the interatomic tunneling ( $i$. e. of the finite bandwidths). Note furthermore that some later results for the macroscopic dielectric function [3, 6, 7] cannot be represented in the form (27). The reason might be in the additional approximations of these works which, in contrast to the present approach, do not include fully the local RPA on-site screening into the dielectric matrix.

In the asymptotic limit of zero bandwidths the expression for $\varepsilon_{M}$ reduces again to the LL form, but with the initial (e. g. Hartree-Fock) values of the dipole matrix element $\mu$ and the energy difference $E_{p}$ replaced by $\left|\mu_{e f f}\right|^{2}=|\mu|^{2} E_{p} / E_{p, e f f}$ and $E_{p, e f f}^{2}=$ $E_{p}^{2}+2 n_{e} E_{p} V_{p, \tilde{p}}(R=0)$ respectively. In the present approach this renormalization is performed within the RPA scheme, $i$. e. the result (27) includes both the crystal and the on-site dielectric screening on the same RPA level. It is appropriate to remind here that with vanishing bandwidths such approach is valid only if the interactions are small with respect to $E_{p}$. It can be also noted that Adler [2] uses the term "selfpolarization correction" for the on-site contributions to the dielectric screening even in the zero-bandwidth limit.

The inclusion of the intra-atomic Coulomb interactions does not alter the previous result (23) concerning the common instability of the transverse and longitudinal modes in narrow band conductors. E. $g$, in the asymptotic limit of zero bandwidths we get, after the inclusion of the on-site "exchange" Coulomb matrix elements into the RPA calculation, both the collective excitations and the macroscopic dielectric function of an atomic insulator with the RPA values of the intra-atomic parameters. If the latter 
represent an appropriate description of the on-site correlations, our method is already fully self-consistent. In the case of the strong on-site correlations, the short-range contributions to the dielectric response have to be treated beyond RPA.

Finally, in the limit of large frequencies $(\omega \rightarrow \infty)$ the macroscopic dielectric function (24) reduces to the simple sum of intra-band and inter-band polarizabilities, with the standard free electron mass expression for the plasma edge [1].

\section{Conclusion}

In conclusion, we point out the main advantages of the present TB approach to the dielectric response. Unlike the representation via the reciprocal lattice indices, it leads to a simple and physically transparent expression for the dielectric matrix, which is explicitly resolved in the particular example of the cubic symmetry. It also clearly distinguishes between the long-range and the local (on-site) contributions to the dielectric screening, and gives so the direct insight into the origin of the self-polarization effects. Furthermore, the present method not only interpolates between (multiband) conductors and insulators, but also includes systems with negligible bandwidths as a well-defined asymptotic limit. Just in this limit we discover an important property of the dielectric instability in multiband conductors, namely that it proceeds by a simultaneous softening of one longitudinal and one transverse collective mode.

In summary, the approach proposed here facilitates the detailed analysis of the dielectric response in crystals. It is particularly efficient in the long wavelength limit, 
even if a large number of bands has to be retained in the calculations. In this respect the decisive criterion for the reducibility of the problem to a finite number of linear equations is the smallness of the on-site exchange terms (25) in comparison with the corresponding long range contributions. Other on-site terms are not relevant (but they have to be weak enough in order to justify the starting RPA scheme). On the other hand, it is clear that our approach has a wide range of applications to real and model systems in which it is appropriate to take into account a small number of bands. 


\section{References}

[1] D. Pines, Elementary Excitations in Solids, W.A. Benjamin, Inc. New York, Amsterdam, 1964; D.Pines and P. Nozieres, The Theory of Quantum Liquids, Vol.1, Addison-Wesley Publishing Co., Inc., 1989.

[2] S.L. Adler, Phys. Rev. 126, 413 (1962).

[3] N. Wiser, Phys. Rev. 129, 62 (1963).

[4] S.G. Louie, J.R. Chelikowsky and M.L. Cohen, Phys. Rev. Letters 34, 155 (1975).

[5] M.S. Hybertsen and S.G. Louie, Phys. Rev. B35, 5585 (1987).

[6] Y. Onodera, Prog. Theor. Physics 49, 37 (1973).

[7] S.K. Sinha, R.P. Gupta and D.L. Price, Phys. Rev. B9, 2654 (1974).

[8] S. Barišić, Phys. Rev. B5, 932 and 941 (1972).

[9] W. Hanke, Phys. Rev. B8, 4585 and 4591 (1973).

[10] For more details see the review article by W. Hanke, Adv. Phys. 27, 287 (1978).

[11] P. Nozieres and D. Pines, Phys. Rev. 109, 741 and 762 (1958) ; Il Nuovo Cimento 9, 470 (1958).

[12] J.A..Van Vechten, R.P. Martin Phys. Rev. Lett. 28, 446 (1972)

[13] P. Županović, A. Bjeliš and S. Barišić (to be published).

[14] M.H. Cohen and F. Keller, Phys. Rev. 99, 1128 (1955). 
[15] For simplicity, we assume that the Fermi level crosses only one set of degenerate bands (i. e. not more than three bands in a cubic latice). The generalization to an accidental crossing of more sets with different symmetries is straightforward.

[16] L. D. Landau and E. M. Lifshitz, Quantum Mechanics (Non-Relativistic Theory), Pergamon Press, 1980.

[17] R.S. Knox, Theory of Excitons, Academic Press, New York (1963).

[18] Note that the Wannier excitons, i. e. the discrete bound states at the edges of interband electron-hole continua, follow from the inclusion of the extended RPA (ladder) terms in the eq.(33) [see also W. Hanke and L.J. Sham, Phys. Rev. B12, 4501 (1975)]. The straightforward generalization of the present approach in this direction shows that the dipolar collective modes and the Wannier excitons can coexist [13].

[19] P.W. Anderson, Concepts in Solids, W.A. Benjamin, Inc. New York, Amsterdam, 1964. 